\title{
C-SEPARATED SETS IN CERTAIN METRIC SPACES
}

\author{
R. F. DICKMAN, JR., R. A. McCOY AND L. R. RUBIN
}

\begin{abstract}
A space $X$ has Property $\mathrm{C}$ provided that for every separated closed subset $A$ of $X$ there exist disjoint closed and connected sets $C_{1}$ and $C_{2}$ in $X$ each of which intersects $A$ and which contain $A$ in their union. This property has been used in characterizing unicoherence. A metric space $X$ has Property $S$ if for each $\varepsilon>0, X$ is the union of a finite number of connected sets each of diameter less than $\varepsilon$. In this paper a sufficient condition for a space to have Property $C$ is established and used to show that separable Hilbert space has Property $\mathrm{C}$ and that every connected metric space having Property $\mathrm{S}$ has Property $\mathrm{C}$. It follows from the latter result that every separable, locally connected, connected, rimcompact metric space has Property C. An example is given of a unicoherent, connected, uniformly locally connected, locally arcwise connected, separable metric space that does not have Property C.
\end{abstract}

The first author defined the concept of a space having Property $\mathrm{C}$ in [2], and in that paper proved that if $X$ is a locally connected, connected normal space which has Property $\mathrm{C}$, then $X$ is unicoherent if and only if every pair of disjoint continua in $X$ can be separated by a connected subset of $X$. This raises the question as to which locally connected, connected normal spaces have Property C. In [3], two classes of spaces were shown to have Property C: all locally compact, locally connected, connected, paracompact, Hausdorff spaces, and all connected polyhedra. In this paper we establish that certain other classes of spaces have Property $C$. However, at the end of the paper we give an example showing that even a unicoherent, connected, uniformly locally connected, locally arcwise connected, separable metric space need not have Property $\mathrm{C}$.

A subset $A$ of a topological space $X$ is $C$-separated provided there exist disjoint closed and connected sets $C_{1}$ and $C_{2}$ of $X$ such that $A \subset\left(C_{1} \cup C_{2}\right)$, $A \cap C_{1} \neq \varnothing$, and $A \cap C_{2} \neq \varnothing$. A space $X$ has Property $C$ if every separated closed subset is $C$-separated.

Lemma. Suppose $A$ and $B$ are disjoint, nonempty, closed subsets of $a$ connected space $X$ and $\mathscr{F}$ is a star finite, locally finite, closed cover of

Received by the editors October 2, 1972.

AMS (MOS) subject classifications (1970). Primary 54F15; Secondary 54D99.

Key words and phrases. $C$-separated, Property C, Property S, Hilbert space manifold.

(c) American Mathematical Society 1973 
$X$ such that $\operatorname{St}(A, \mathscr{F}) \cap \operatorname{St}(B, \mathscr{F})=\varnothing$. Then $A \cup B$ is $C$-separated if either:

1. Each element of $\mathscr{F}$ is connected and locally connected and $X$ is regular, or

2. Each element of $\mathscr{F}$ is arcwise connected and X is Hausdorff.

Proof. Let $C$ be a component of $\operatorname{St}(A \cup B, \mathscr{F})$. Since $\mathscr{F}$ is locally finite, $C$ is closed in $X$. Also $C$ does not contain $A \cup B$, although $C \cap$ $(A \cup B) \neq \varnothing$. Let $C_{0}=\operatorname{St}(A \cup B, \mathscr{F}) \backslash C$, which is closed in $X$. Let $C_{1}$ be the union of members of $\mathscr{F}$ which intersect $C_{0}$ but not $C$. By induction define $C_{n+1}$ to be the union of members of $\mathscr{F}$ which intersect $C_{n}$ but not $C$. Then set $D=\bigcup_{n=0}^{\infty} C_{n}$, so that $C$ and $D$ are disjoint, $A \cup B \subset C \cup D$, $(A \cup B) \cap C \neq \varnothing$, and $(A \cup B) \cap D \neq \varnothing$.

For each component $D^{\prime}$ of $D$, there is an $F\left(D^{\prime}\right) \in \mathscr{F}$ such that $F\left(D^{\prime}\right) \cap$ $C \neq \varnothing$ and $F\left(D^{\prime}\right) \cap D \neq \varnothing$. Let $\left\{D_{0}, D_{1}, \cdots, D_{\beta}, \cdots\right\}, \beta<\alpha$, be a wellordering on the set of components of $D$.

We shall define by transfinite induction two sets of continua,

$$
\left\{K_{1}, K_{2}, \cdots, K_{\beta}, \cdots\right\} \text { and }\left\{L_{1}, L_{2}, \cdots, L_{\beta}, \cdots\right\}, \quad \beta<\alpha .
$$

Let $\left\{D_{\beta_{1}}, \cdots, D_{\beta_{m}}\right\}$ be the set of components of $D$ which intersect $F\left(D_{1}\right)$, which is finite because $\mathscr{F}$ is star finite. Since $F\left(D_{1}\right)$ is either locally connected or arcwise connected, there exists a closed subset $F_{1}$ of $F\left(D_{1}\right)$ such that $C \cup D_{0} \cup F_{1} \cup D_{\beta_{1}} \cup \cdots \cup D_{\beta_{m}}$ has two components, say $K_{1}$ containing $C$ and $L_{1}$ containing $D_{0}$ [3, Lemma 8]. Observe that if $\beta<\alpha$ and $D_{\beta} \notin\left\{D_{\beta_{1}}, \cdots, D_{\beta_{m}}\right\}$, then $D_{\beta} \cap\left(K_{1} \cup L_{1}\right)=\varnothing$; otherwise $D_{\beta} \subset K_{1} \cup L_{1}$.

Now suppose $\beta<\alpha$ and that for each $\gamma<\beta$, sets $K_{\gamma}$ and $L_{\gamma}$ have been defined so that:

1. $K_{\gamma}$ and $L_{\gamma}$ are disjoint continua with $C \subset K_{\gamma}$ and $D_{0} \subset L_{\gamma}$.

2. If $\delta<\gamma$, then $K_{\delta} \subset K_{\gamma}$ and $L_{\delta} \subset L_{\gamma}$.

3. $K_{\gamma} \cup L_{\gamma}$ is the union of members of $\left\{D_{0}, D_{1}, \cdots, D_{\delta}, \cdots\right\}$ and one closed subset $F_{\delta}$ of $F\left(D_{\delta}\right)$ for each $\delta \leqq \gamma$.

4. $K_{\gamma} \cup L_{\gamma}$ contains all $D_{\delta}$ for $\delta \leqq \gamma$, and for any $\delta<\alpha, D_{\delta}$ intersects $K_{\gamma} \cup L_{\gamma}$ if and only if $D_{\delta}$ is a subset of $K_{\gamma} \cup L_{\gamma}$.

Let $K^{\prime}=\bigcup_{\gamma<\beta} K_{\gamma}$ and $L^{\prime}=\bigcup_{\gamma<\beta} L_{\gamma}$. Then $K^{\prime}$ and $L^{\prime}$ are disjoint continua such that $C \subset K^{\prime}$ and $D_{0} \subset L^{\prime}$. If $D_{\beta}$ intersects $K^{\prime} \cup L^{\prime}$, then set $K_{\beta}=K^{\prime}, L_{\beta}=L^{\prime}$, and $F_{\beta}=\varnothing$. Then $D_{\beta} \subset K_{\beta} \cup L_{\beta}$ and the conditions 1 through 4 are satisfied. If $D_{\beta}$ does not intersect $K^{\prime} \cup L^{\prime}$, let $\left\{D_{\beta_{1}}, \cdots, D_{\beta_{n}}\right\}$ be the components of $D$ which intersect $F\left(D_{\beta}\right)$. Note that $F\left(D_{\beta}\right) \cap K^{\prime} \neq \varnothing$. Again by [3, Lemma 8], there exists a closed subset $F_{\beta}$ of $F\left(D_{\beta}\right)$ such that $K^{\prime} \cup L^{\prime} \cup F_{\beta} \cup D_{\beta_{1}} \cup \cdots \cup D_{\beta_{n}}$ has two components, $K_{\beta}$ and $L_{\beta}$, with $K^{\prime} \subset K_{\beta}$ and $L^{\prime} \subset L_{\beta}$. Thus the conditions 1 through 4 are satisfied in this case also.

Finally, let $K=\bigcup_{\beta<\alpha} K_{\beta}$ and $L=\bigcup_{\beta<\alpha} L_{\beta}$. Then $K$ and $L$ are disjoint 
continua, $A \cup B \subset K \cup L,(A \cup B) \cap K \neq \varnothing$, and $(A \cup B) \cap L \neq \varnothing$. Therefore $A \cup B$ is $C$-separated.

A metric space $X$ has Property $\mathrm{S}$ provided that, for each $\varepsilon>0, X$ is the union of a finite number of connected sets each of diameter less than $\varepsilon$. This property is discussed in [7] and is shown to imply local connectedness.

TheOREM 1. Every connected metric space having Property S, has Property C.

Proof. Let $A$ and $B$ be disjoint, nonempty, closed subsets of $X$, where $X$ is a connected metric space, with metric $d$, having Property $\mathbf{S}$. We shall construct a cover $\mathscr{F}$ of $X$ satisfying the hypothesis of the lemma. By [7, Corollary 15.41], there exists a finite open cover $\mathscr{G}_{1}$ of $X$ by connected sets having Property $S$ such that mesh $\mathscr{G}_{1} \leqq \frac{1}{2}$. Then by induction, we can obtain a sequence $\left\{\mathscr{G}_{i}\right\}_{i=1}^{\infty}$ of finite open covers of $X$ such that for each $n, \mathscr{G}_{n+1}$ refines $\mathscr{G}_{n}$, mesh $\mathscr{G}_{n} \leqq 1 / 2^{n}$, and the members of $\mathscr{G}_{n}$ are connected sets having Property $\mathrm{S}$.

Setting $H_{0}=\varnothing$ and using induction again, we can define sequences $\left\{\mathscr{D}_{i}\right\}_{i=1}^{\infty}$ and $\left\{H_{i}\right\}_{i=0}^{\infty}$ so that, for each $n$,

$$
\mathscr{D}_{n}=\left\{G \in \mathscr{G}_{n} \mid\left(d(G, A)>\frac{1}{2^{n-1}} \text { or } d(G, B)>\frac{1}{2^{n-1}}\right)\right.
$$

$$
\text { and } \left.G \cap\left(X \backslash \bigcup_{i=0}^{n-1} H_{i}\right) \neq \varnothing\right\}
$$

and

$$
H_{n}=\bigcup\left\{D \mid D \in \mathscr{D}_{n}\right\} \text {. }
$$

Then let $\mathscr{D}=\bigcup_{i=1}^{\infty} \mathscr{D}_{i}$ and $\mathscr{F}=\{\bar{D} \mid D \in \mathscr{D}\}$. The members of $\mathscr{F}$ are connected and have Property S, so that they are also locally connected.

To see that $\mathscr{D}$, and hence $\mathscr{F}$, is a cover of $X$, suppose that $x \notin \bigcup_{i=1}^{\infty} H_{i}$. Then whenever $x \in G \in \mathscr{G}_{n}, d(G, A) \leqq 1 / 2^{n-1}$ and $d(G, B) \leqq 1 / 2^{n-1}$. This implies that $x$ is a limit point of both $A$ and $B$, which is impossible since $A$ and $B$ are disjoint.

To see that $\mathscr{F}$ is star finite, let $F \in \mathscr{F}$. Then $F=\bar{D}$, where $D \in \mathscr{D}_{n}$ for some $n$. We may suppose that $d(D, A)>1 / 2^{n-1}$. Let $m>n+2$, and let $D^{\prime} \in \mathscr{D}_{m}$. In order to show that $\bar{D} \cap \bar{D}^{\prime}=\varnothing$, it suffices to show that $d(x, D)>1 / 2^{n+1}$ for every $x \in D^{\prime} \backslash \bigcup_{i=1}^{m-1} H_{i}$. So let $x \in D^{\prime} \backslash \bigcup_{i=1}^{m-1} H_{i}$, and assume that $d(x, D) \leqq 1 / 2^{n+1}$. Suppose $x \notin \bigcup_{i=1}^{n} H_{i}$. There exists $G \in \mathscr{G}_{n+1}$ such that $x \in G$. Now $d(x, A)>3 / 2^{n+1}$, so that since diam $G<1 / 2^{n+1}$, then $d(G, A)>1 / 2^{n}$. Hence $G \in \mathscr{D}_{n+1}$, so that $x \in H_{n+1}$. Thus in any case, $x \in \bigcup_{i=1}^{n+1} H_{i} \subset \bigcup_{i=1}^{m-1} H_{i}$-which is a contradiction. 
The local finiteness of $\mathscr{F}$ follows from the star finiteness since the members of $\mathscr{F}$ are closures of open sets which themselves cover $X$.

Finally it remains to establish that $\operatorname{St}(A, \mathscr{F}) \cap \operatorname{St}(B, \mathscr{F})=\varnothing$. Suppose there are $F_{1}, F_{2} \in \mathscr{F}$ such that $x \in F_{1} \cap F_{2}, F_{1} \cap A \neq \varnothing$, and $F_{2} \cap B \neq \varnothing$. There exist $D_{m} \in \mathscr{D}_{m}$ and $D_{n} \in \mathscr{D}_{n}$ such that $F_{1}=\overline{D_{m}}$ and $F_{2}=\overline{D_{n}}$. Now $1 / 2^{n-1} \leqq d(x, A) \leqq 1 / 2^{m}$, so that $m<n$. But also $1 / 2^{m-1} \leqq d(x, B) \leqq 1 / 2^{n}$, so that $n<m$. This is impossible, so that $\operatorname{St}(A, \mathscr{F}) \cap \operatorname{St}(B, \mathscr{F})=\varnothing$ as desired.

Corollary 1. If $X$ is a separable, locally connected, connected, rimcompact metric space, then $X$ has Property $\mathrm{C}$.

Proof. We need to show that $X$ has a Property $\mathrm{S}$ metric. Let $\gamma X$ denote the Freudenthal compactification of $X$, and let $\varepsilon>0$. By [5, Theorem 5] $\gamma X$ is a metric space, say with metric $d$. By [5, Theorem 1], there exists a finite covering $\left\{U_{1}, \cdots, U_{n}\right\}$ of $\gamma X$ by connected open sets such that for each $i$, diam $U_{i}<\varepsilon$ and the boundary of $U_{i}$ is contained in $X$. By [6, Lemma 5], $V_{i}=U_{i} \cap X$ is connected for each $i$. Thus $\left\{V_{1}, \cdots, V_{n}\right\}$ is a finite open cover of $X$ by connected sets whose diameters are less than $\varepsilon$, and so $d \mid X$ is a Property S metric for $X$.

COROLlaRY 2. If $X$ is a locally compact, locally connected, connected metric space, then $X$ has Property $\mathrm{C}$.

This second corollary also follows from results in [3], which we have stated in our introductory paragraph. The rimcompactness in Corollary 1 is not a necessary condition since Hilbert space $l_{2}$ has Property $\mathrm{C}$, as seen from the next theorem. The fact that $l_{2}$ is not rimcompact can be easily seen from the fact that $l_{2} \backslash A$ is homeomorphic to $l_{2}$ for any compact subset $A$ of $l_{2}$ [4]. By a Hilbert space manifold is meant a separable metric space such that each point has a neighborhood homeomorphic to $l_{2}$.

TheOREM 2. Every connected Hilbert space manifold has Property C.

Proof. Let $M$ be a connected Hilbert space manifold, and let $A$ and $B$ be disjoint nonempty closed subsets of $M$. Let $U$ and $V$ be disjoint open subsets of $M$ such that $A \subset U$ and $B \subset V$. Let $\mathscr{W}=\{U, V, M \backslash(A \cup B)\}$. By [1], there exists a countable, locally finite simplicial complex $K$ and a homeomorphism $h$ from $M$ onto $|K| \times l_{2}$ such that for each simplex $\sigma \in K$, $|\sigma| \times l_{2} \subset h(W)$ for some $W \in \mathscr{W}$. Let $\mathscr{F}=\left\{h^{-1}\left(|\sigma| \times l_{2}\right) \mid \sigma \in K\right\}$, which is a star finite, locally finite closed cover of $M$ by sets which are connected and locally connected. Since $\mathscr{F}$ is a refinement of $\mathscr{W}, \operatorname{St}(A, \mathscr{F}) \cap$ $\operatorname{St}(B, \mathscr{F})=\varnothing$. Then by the lemma, $M$ has Property C.

A metric space $X$ is uniformly locally connected provided that for each $\varepsilon>0$, there exists a $\delta>0$ such that every two points $x$ and $y$ of $X$ whose 
distance apart is less than $\delta$ lie together in a connected subset of $X$ of diameter less than $\varepsilon$. This property is discussed in [7], and, like Property $\mathrm{S}$, it also implies local connectedness.

We now give an example of a unicoherent connected, uniformly locally connected, locally arcwise connected, separable metric space $Y$ which does not have Property C.

Let $Q$ denote the set of rational numbers in the closed interval $I=[0,1]$. Let $X=\prod_{1}^{\infty} I$; if $x \in X$, write $x=\left(x_{1}, x_{2}, \cdots\right)$. Let $F_{1}=\left\{x \in X \mid x_{i}=0\right.$ for $i>1\}$. For $n>1$, let $F_{n}=F_{n-1} \cup\left\{x \in X \mid x_{k} \in Q\right.$ for $1 \leqq k \leqq n-1$ and $x_{k}=0$ for $k>n\}$. Let $Y=\bigcup_{i=1}^{\infty} F_{i}$. We construct a metric $d$ for $Y$ by induction. If $x, z \in F_{1}$, then define $d(x, z)=\left|x_{1}-z_{1}\right|$. If $d$ has been given for $F_{n-1}$, let $x, z \in F_{n}$. If $x_{1}=z_{1}, x_{2}=z_{2}, \cdots, x_{n-1}=z_{n-1}$, then define $d(x, z)=$ $\left|x_{n}-z_{n}\right|$. Otherwise $x^{\prime}=\left(x_{1}, x_{2}, \cdots, x_{n-1}, 0,0, \cdots\right)$ and $z^{\prime}=\left(z_{1}, z_{2}, \cdots\right.$, $\left.z_{n-1}, 0,0, \cdots\right)$ are in $F_{n-1}$ and we define $d(x, z)=x_{n}+z_{n}+d\left(x^{\prime}, z^{\prime}\right)$. It is easy to check that this is an extension of the metric on $F_{n-1}$ and that $d$ provides a metric for $Y$. We shall prove $Y$ does not have Property $\mathrm{C}$, but we leave it to the reader to establish that $Y$ has all the other properties mentioned above.

For each $n=2,3, \cdots$, let $K_{n}=\left\{x \in F_{n} \mid x_{n}=1\right\}$. One can check that each $K_{n}$ is closed in $Y$, and, moreover, $K=\bigcup_{n=2}^{\infty} K_{n}$ is closed in $Y$. Clearly $K$ is disconnected. We shall indicate why $K$ cannot be $C$-separated in $Y$.

Let $L$ and $M$ be continua of $Y$ which $C$-separate $K$. First we show that $F_{1} \subset L \cup M$. For suppose $F_{1} \not L \cup M$. Then there is an $x \in F_{1}$ such that $x_{1} \in Q$ and $x \notin L \cup M$. Let $\bar{x}=\left(x_{1}, 1,0,0, \cdots\right) \in K_{2}$. Then we can write $Y-\{x\}$ as the disjoint union of two open sets $U$ and $V$ with $\bar{x} \in U$ and $K_{2}-\{\bar{x}\} \subset V$. Thus we may as well assume $K_{2}-\{\bar{x}\} \subset L \subset V$ and $\bar{x} \in M \subset U$. But for any $t_{1} \in Q$ such that $t_{1} \neq x_{1}$, the point $t=\left(t_{1}, 0,0, \cdots\right)$ is in $F_{1}$ and separates two points of $K_{2}-\{\bar{x}\}$, implying $t \in L$. But this implies $x \in L$ as well because there is a sequence of such points $t \in F_{1}$ converging to $x$. This proves $F_{1} \subset L \cup M$.

We next show $F_{2} \subset L \cup M$. To do this, let $x_{1} \in Q$ and let $G_{2}=\left\{z \in F_{2} \mid\right.$ $\left.z_{1}=x_{1}\right\}$. Note that $G_{2}$ is an arc. We shall prove $G_{2} \subset L \cup M$. Suppose this is not true. Then there is an $x \in G_{2}$ such that $x_{2} \in Q$ and $x \notin L \cup M$. Let $\bar{x}=\left(x_{1}, x_{2}, 1,0,0, \cdots\right)$, which is in $K_{3}$. The proof that $G_{2} \subset L \cup M$ now proceeds as in the proof that $F_{1} \subset L \cup M$. Since $F_{2}$ is a union of sets $G_{2}$, then $F_{2} \subset L \cup M$. Using induction and an obvious extension of the above approach, we obtain the result that $F_{i} \subset L \cup M$ for all $i$. But then, $Y=\bigcup_{i=1}^{\infty} F_{i}$ is disconnected, a contradiction. Therefore $Y$ does not have Property C.

REMARK. Every connected, locally connected metric space has a compatible metric which is uniformly locally connected. This result can be found in [7, p. 154]. On the other hand, the above example and 
Theorem 1 show that not every connected, locally connected, separable metric space has a compatible metric which has Property $\mathrm{S}$.

\section{REFERENCES}

1. W. H. Cutler, A triangulation theorem for F-manifolds, General Topology and Appl. 2 (1972), 45-48.

2. R. F. Dickman, Jr., Some mapping characterizations of unicoherence, Fund. Math. 78 (1973), 27-35.

3. R. F. Dickman, Jr., and L. R. Rubin, C-separated sets and unicoherence, Fund. Math. (to appear).

4. V. L. Klee, Jr., A note on topological properties of normed linear spaces, Proc. Amer. Math. Soc. 7 (1956), 673-674. MR 17, 1227.

5. K. Morita, On bicompactifications of a semicompact space, Sci. Rep. Tokyo Bunrika Daigaku Sect. A 4 (1952), 222-229. MR 14, 571.

6. - On images of an open interval under closed continuous mappings, Proc. Japan Acad. 32 (1956), 15-19. MR 18, 140.

7. G. T. Whyburn, Analytic topology, Amer. Math. Soc. Colloq. Publ., vol. 28, Amer. Math. Soc., Providence, R.I., 1942. MR 4, 86.

Department of Mathematics, Virginia Polytechnic Institute and State UNIVERSITY, BLACKSBURG, VIRGINIA 24061

Department of Mathematics, University of Oklahoma, Norman, Oklahoma 73069 